Pacific Journal of Mathematics

A THEOREM ON FAMILIES OF ACYCLIC SETS AND ITS 


\title{
A THEOREM ON FAMILIES OF ACYCLIC SETS AND ITS APPLICATIONS
}

\author{
A. Kosinski
}

In the first part of this note we discuss a group of theorems dealing with geometric configurations arising when we assign in continuous way compact, acyclic sets to $k$-planes in the Euclidean $n$-dimensional space $E_{n}$. A fairly representative example of those theorems is as follows:

Suppose that to every (unoriented) $k$-plane $H$ through a point $a$ of $E_{n}$ there is upper semi-continuously assigned a compact and acyclic set $\Phi(H) \subset H$. Then for some plane $H_{0}, a \in \Phi\left(H_{0}\right)$.

In fact, we will prove a much more general theorem of which the above is one of the consequences.

In the second part of this note we give various applications of the above theorems. They are related to the theory of convex sets $(\S 2.1-2.4)$, mappings of manifolds $(\S 2.6)$, and to some relations between vector fields and involutions on $S_{n}(\S 2.5)$.

The author wishes to acknowledge his indebtedness to Dr. M. Hirsch for valuable suggestions and to Dr. J. W. Jaworowski whose generalization of the author's previous results was the starting point for the present paper.

\section{Families of compact sets over Grassmannians.}

1.1 $H_{n}(X)$ will denote the $n$th Cech homology group of the space $X$ with the group $Z_{2}$ of integers mod 2 as the group of coefficients. We will say that $X$ is acyclic if $X$ is connected and $H_{n}(X)=0, n=1,2, \cdots$, ,

Let $X$ be a compact metric space and let $\Phi: X \rightarrow 2^{Y}$ be an upper semi-continuous mapping of $X$ into the space $2^{Y}$ of all nonempty compact subsets of a space $Y$. The triple $\mathscr{F}=\{X, Y, \Phi\}$ will be called a family [3]. The set $X$ will be called the basis of $\mathscr{F}$, the sets $\Phi(x)$ the elements of $\mathscr{F}$, the set $\bigcup_{x \in X} \Phi(x) \subset Y$ - the field of $\mathscr{F}$. The field will be also denoted $\Phi(X)$. A family $\mathscr{F}$ is said to be acyclic if all its elements are acyclic.

If $\mathscr{F}=\{X, Y, \Phi\}$ is a family then the subset $M=\{(x, y) \mid y \in \Phi(x)\}$ of the cartesian product $X \times Y$ is called the graph of $\mathscr{F}$.

$M$ is a closed subset of $X \times Y$ (and, hence, compact) because of the upper semi-continuity of $\Phi$, and this is the only reason for requiring the upper semi-continuity of $\Phi$.

$G_{p, q}$ will denote the Grassmannian of (unoriented) $q$-planes through

Received January 27, 1961. Presented to the American Mathematical Society, April 8, 1961. Received by the Editors January 27, 1961. Supported in part by the National Science Foundation under Grant G 10700. 
the origin of the $p$-dimensional Euclidean space $E_{p}$.

Let $T$ be a fixed $k$-plane in $E_{n}$ and let $r>k$. Let $E_{n-k}$ be the Euclidean space orthogonal to $T$ in $E_{n}$ and for $x \in G_{n-k, r-k}$ let $H(x)$ be the $r$-plane in $E_{n}$ spanned by $x$ and $T$. The correspondence $x \rightarrow H(x)$ is obviously one-to-one correspondence between $G_{n-k, r-k}$ and the set of all $r$-planes in $E_{n}$ containing $T$. Henceforth we will say that $H(x)$ is the $r$-plane corresponding to $x$.

1.2. Theorem. Let $T$ be a fixed k-plane in $E_{n}$. For every $x \in G_{n-k, r-k}$ let $H(x)$ be the r-plane in $E_{n}$ containing $T$ and corresponding to $x$.

If $\mathscr{F}=\left\{G_{n-k, r-k}, E_{n}, \Phi\right\}$ is an acyclic family then there exists an $x \in G_{n-k, r-k}$ such that $H(x) \cap \Phi(x) \neq 0$.

Proof. Let us remark first that it is enough to prove the theorem in case $r=k+1$. For in a general case we may always choose an $(r-1)$-plane $T_{0}$ containing $T$ and the Grassmannian of all $r$-planes containing $T_{0}$ is a subset of $G_{n-k, r-k}$.

Therefore we will assume that $r=k+1$, i.e., the basis of $\mathscr{F}$ is the set of all $(k+1)$-planes containing a given $k$-plane $T$. Then

$$
G_{n-k, r-k}=G_{n-k, 1}=P_{n-k-1}
$$

and we may write $\mathscr{F}=\left\{P_{n-k-1}, E_{n}, \Phi\right\}$, where $P_{n-k-1}$ denotes the $(n-$ $k-1$ )-dimensional projective space.

Let $S$ be an $(n-1)$-sphere in $E_{n}$ with center $o$ in $T$ and containing the field of $\mathscr{F}$ in the interior. Since the field of $\mathscr{F}$ is compact such a sphere exists. Let $\Phi_{1}(x)=H(x) \cap S$ for every $x \in P_{n-k-1}$. Thus $\Phi_{1}(x)$ is a $k$-dimensional great circle in $S$ and $\mathscr{F}_{1}=\left\{P_{n-k-1}, E_{n}, \Phi_{1}\right\}$ is a family.

Let $J \subset P_{n-k-1} \times E_{n} \times I \times E_{n}, \quad I=\langle 0,1\rangle$, be the union of all sets of the form

. $(x) \times \Phi(x) \times I \times \Phi_{1}(x), x \in P_{n-k-1}$;

i.e., $\left(x, y, t, y_{1}\right) \in J$ if and only if $y \in \Phi(x), y_{1} \in \Phi_{1}(x), t \in\langle 0,1\rangle$.

We shall identify in $J$

$$
\left(x, y, 1, y_{1}\right) \quad \text { with } \quad\left(\bar{x}, \bar{y}, 1, \bar{y}_{1}\right) \quad \text { if } \quad x=\bar{x}, y=\bar{y}
$$

and

$$
\left(x, y, 0, y_{1}\right) \text { with }\left(\bar{x}, \bar{y}, 0, \bar{y}_{1}\right) \quad \text { if } \quad x=\bar{x}, y_{1}=\bar{y}_{1} .
$$

Denote the set obtained from $J$ by these identifications by $M$. Then $M$ is the union of sets of the form $(x) \times \Phi(x) * \Phi_{1}(x)$ where $x \in P_{n-k-1}$ 
and $A * B$ is the notation for the join ${ }^{1}$ of $A$ with $B$. It is easy to see that because of the upper semi-continuity of $\Phi M$ is compact.

Let $M_{0} \subset M$ be composed of points with $t=0$; more precisely, $M_{0}$ is the image under the identification mapping $J \rightarrow M$ of the subset of $J$ composed of points of the form $\left(x, y, 0, y_{1}\right)$. Let $q: M_{0} \rightarrow S$ and $p: M_{0} \rightarrow$ $P_{n-k-1}$ be defined by $q\left(x, y, 0, y_{1}\right)=y_{1}, p\left(x, y, 0, y_{1}\right)=x$. Then

(i ) $M_{0}$ is an $(n-1)$-manifold, and $q$ is a mapping of degree 1 . If $k=0$ then $p$ is a covering map. In particular, for every $k \geqq 0$ $p_{*} H_{n-1}\left(M_{0}\right)=0$ and $q_{*} H_{n-1}\left(M_{0}\right)=H_{n-1}(S)$.

For let $M_{1}$ be the graph of $\Phi_{1}$, i.e. the set of points $(x, z) \subseteq P_{n-k-1} \times E_{n}$ satisfying $z \in \Phi_{1}(x)$. Let $p_{1}: M_{1} \rightarrow P_{n-k-1}$ and $q_{1}: M_{1} \rightarrow S$ be defined by $p_{1}(x, z)=x, q_{1}(x, z)=z$ and let $h: M_{0} \rightarrow M_{1}$ be defined by $h\left(x, y, 0, y_{1}\right)=$ $\left(x, y_{1}\right)$. Then the diagram

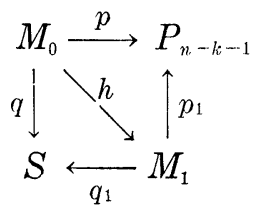

is commutative. Moreover, because of identifications, $h$ is a homeomorphism. But it is easy to see that $M_{1}$ is a fibre space over $P_{n-k-1}$ with the fibre $S_{k}$ and $p_{1}$ as the fibering map. This proves that $M_{0}$ is an $(n-1)$-manifold. Now, since

$$
\Phi_{1}(x) \cap \Phi_{1}(y) \subset T \cap S
$$

if $x \neq y$ it follows that $q_{1}$ maps $q_{1}^{-1}(S-T)$ homeomorphically onto $S-T$. This proves that $q$ is of degree 1 . If $k=0$ then $q_{1}$ is a homeomorphism and $p_{1}$ is a covering map. This proves (i).

Now, let us consider the diagram

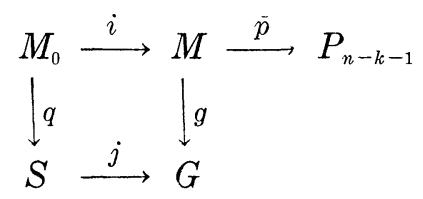

where $i$ and $j$ are inclusion maps, $\bar{p}: M \rightarrow P_{n-k-1}$ is defined by $\bar{p}\left(x, y, t, y_{1}\right)=$ $x$ and $g\left(x, y, t, y_{1}\right)=$ point in $E_{n}$ dividing the segment $\overline{y_{1} y}$ in the ratio $t /(1-t) ; G=g(M)$. Since

$$
g\left(x, y, 0, y_{1}\right)=y_{1}=q\left(x, y, 0, y_{1}\right)
$$

the diagram is commutative.

1 i.e., the set obtained from $A \times I \times B$ by identifying $A \times<0>\times B$ with $A$ and $A \times$ $\langle 1\rangle \times B$ with $B$ by means of the mappings $(x, 0, y) \rightarrow x$ and $(x, 1, y)=y$. 


\section{Observe that}

(ii) $\bar{p}^{-1}(x)$ is acyclic and nonempty for every $x \in P_{n-k-1}$.

For $\bar{p}^{-1}(x)$ is homeomorphic with $\Phi(x) * \Phi_{1}(x)$ and join of an acyclic set with a compact set is acyclic. ${ }^{2}$

Therefore it follows from the Vietoris Mapping Theorem [2] that in the diagram

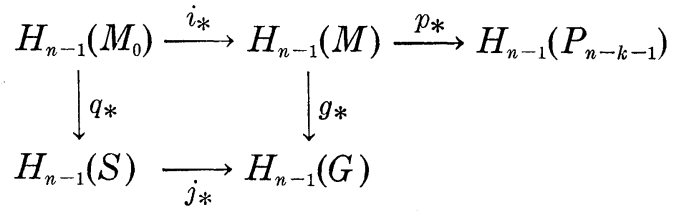

$\bar{p}_{*}$ is an isomorphism onto. But $\bar{p} i=p$ and it follows from (i) that $p_{*}$ $\mathrm{i}^{\mathrm{s}}$ trivial for every $k \geqq 0$. Therefore

(iii) $i_{*}$ is trivial.

Now, again by (i), $q_{*}$ is onto Thus (iii) implies that $j_{*}$ is trivial, i.e., that $S$ bounds in $G$. Therefore $G$ must contain the ball bounded by $S$ and we conclude that the center $o$ of $S$ is in $G$. This means that for some $x \in P_{n-k-1}$ there exist points $y \in \Phi(x), y_{1} \in \Phi_{1}(x)$ such that the segment joining $y_{1}$ with $y$ contains $o$. Since $y_{1} \in H(x)$ and $o \in H(x)$ it follows that $y \in H(x)$, i.e., $y \in \Phi(x) \cap H(x)$. This proves the theorem.

1.3. CoRollaRy. Let $G_{n-k, n-r}$ be the Grassmannian of all $(n-r)$ planes contained in an $(n-k)$-plane $T$. For every $x \in G_{n-k, n-r}$ let $H^{*}(x)$ be the orthogonal complement in $E_{n}$ of the plane representing $x$. Let $\mathscr{F}=\left\{G_{n-k, n-r}, E_{n}, \Phi\right\}$ be an acyclic family. Then there exists an $x \in G_{n-k, n-r}$ such that

$$
\Phi(x) \cap H^{*}(x) \neq 0 .
$$

Proof. Let $T^{*}$ be the orthogonal complement of $T$ in $E_{n}$ and $G_{n-k, r-k}$ the Grassmannian of all $r$-planes in $E_{n}$ containing $T^{*}$. For every $y \in G_{n-k, r-k}$ let $G(y)$ be the plane representing $y$ and $G^{*}(y)$ its orthogonal complement. Then $G^{*}(y) \cap T$ represents an element $x=f(y)$ of $G_{n-k, n-r}$. Moreover it is easily seen that

$$
G(y)=H^{*}(f(y)) \text {. }
$$

Let $\mathscr{F}_{1}=\left\{G_{n-k, r-k}, E_{n}, \Phi(f(y))\right\}$. Then $\mathscr{F}_{1}$ is an acyclic family and by Theorem 1.2 there exists an $y \in G_{n-k, r-k}$ such that $\Phi(f(y)) \cap G(y) \neq 0$. Compared with (i) this gives

2 Short proof. Let $A * B$ be the join of $A$ with $B$. Suppose that $A$ is acyclic and let $f: A * B \rightarrow A * B$ be defined by $f(x, t, y)=\left(x_{0}, t, y\right)$ where $x_{0}$ is a fixed point of $A$. Then $f^{-1}\left(x_{0}, t, y\right)$ is homeomorphic with $A$ if $t \neq 1$ and is a point if $t=1$. In both cases $f^{-1}\left(x_{0}\right.$, $t, y)$ is acyclic and, by Vietoris mapping theorem [2], $f$ induces an isomorphism of $H_{k}(A * B)$ onto $\mathrm{H}_{k}(f(A * B)), k=0,1,2, \cdots$, . But $f(A * B)=\left(x_{0}\right) * B$ is acyclic, therefore $A * B$ is also. 


$$
\Phi(x) \cap H^{*}(x) \neq 0
$$

In the following two corollaries we suppose that a point $p \in E_{n}$ is given and a natural one-to-one correspondence $x \rightarrow H(x)$ between the set of all $k$-planes $H(x)$ through $p$ is fixed once and for all.

1.4. Corollary. Let $\mathscr{F}=\left\{G_{n, k}, E_{n}, \Phi\right\}$ be an acyclic family. Then for some $x_{0} \in G_{n, k}, \Phi\left(x_{0}\right) \cap H\left(x_{0}\right) \neq 0$ and for some $x_{1} \in G_{n, k}, \Phi\left(x_{1}\right) \cap$ $H^{*}\left(x_{1}\right) \neq 0$.

Proof. (The second part of this corollary was first proved in another way by Dr. J.W. Jaworowski.) Existence of $x_{0}$ follows from the Theorem 1.2 with $k=0$; existence of $x_{1}$ follows from the Corollary 1.3 with $r=n-k$.

1.5. Corollary. Let $\mathscr{F}=\left\{G_{n, k}, E_{n}, \Phi\right\}$ be an acyclic family satisfying the condition $\Phi(x) \subset H(x)$ where $H(x)$ is the plane through $p$ representing $x$. Then for some $x, p \in \Phi(x)$.

Proof. By Corollary 1.4, for some $x \Phi(x) \cap H^{*}(x) \neq 0$. Since $\Phi(x) \subset H(x)$ this implies $\Phi(x) \cap H(x) \cap H^{*}(x) \neq 0$ and in view of $p=$ $H(x) \cap H^{*}(x)$ this proves the corollary.

\section{Applications.}

2.1. We start with a simple application of 1.5. Let $A \subset E_{n}$ be a compact subset of $E_{n}$ and $k$ a fixed integer, $1 \leqq k \leqq n-1$. Let $A_{k}$ be the set of such points $p \in E_{n}$ that every $k$-plane $H$ through $p$ intersects $A$ in an nonempty acyclic set. Applying 1.5 to the family $\mathscr{F}=$ $\left\{G_{n, k}, E_{n}, H \cap A\right)$ we infer that $p \in A$, i.e. $A_{k} \subset A$.

In a subsequent paper we will prove that $A$ is star-shaped with respect to every point of $A_{k}$, i.e. if $x \in A$ and $p \in A_{k}$ then the segment $\overline{x p} \subset A$. In particular, it follows $A_{k}=A_{k+1}, k=1, \cdots, n-2$.

2.2. The following is a generalization of a theorem of H. Steinhaus [8] (see also S.K. Stein [7]).

Theorem. Let $A$ be a convex subset of $E_{n}$ and $p \in \operatorname{Int} A$. Then there exists a sequence of planes $H_{1}, H_{2}, \cdots, H_{n-1}$ such that $H_{i}$ is an $i$-plane, $H_{i} \subset H_{i+1} i=1, \cdots, n-2$, and $p$ is the center of gravity of $A \cap H_{i}, i=1,2, \cdots, n-1$.

Proof. For every $k$-plane $H$ through $p$ let $x(H)$ be the center of 
gravity of $H \cap A$. Then our assumptions about $A$ insure that $\mathscr{F}=$ $\left\{G_{n, k}, E_{n}, x(H)\right\}$ is a family. Therefore it follows from 1.5 that $x(H)=p$ for some $H$. Thus to prove the theorem we start with $k=n-1$ and find the $(n-1)$-plane $H_{n-1}$ such that $x\left(H_{n-1}\right)=p$. Then in $A \cap H_{n-1}$, which is again convex and $p \in \operatorname{Int} A \cap H_{n-1}$, we find $H_{n-2}$, and so we continue until the sequence is complete.

We may remark that the assumption that $A$ is convex and $p \in \operatorname{Int} A$ are needed only to insure that $x(H)$ is a continuous function of $H$. Also we may suppose that a continuous positive density is given on $A$. The theorem may be correspondingly generalized without a change in proof.

2.3. The following theorem, first proved by G. Aumann [1], is an easy consequence of 1.5 .

THEOREM. Let $M$ be a compact subset of $E_{n}$ and suppose that for some $k \geqq 1$ intersection of every $k$-plane with $M$ is either empty or acyclic. Then $M$ is convex.

This is a consequence of the following lemma which we will prove first:

Lemma. Let $M$ be a compact subset of $E_{n}$ and suppose that some $k$-plane $H$ intersects the convex hull of $M$ but does not intersect $M$. Then there exists a k-plane $H_{0}$ such that $M \cap H_{0}$ is not connected.

Proof. Let $p, q \in M$, let $r$ be a point of the segment $\overline{p q}$ and let $H$ be a $k$-plane in $E_{n}$ such that $H \cap \overline{p q}=r$ and $H \cap M=0$. Let $H^{\prime}$ be the $(k+1)$-dimensional plane in $E_{n}$ containing $H$ and the line through $p, q$. Then $H$ disconnects $H^{\prime}$ between $p$ and $q$. Thus if $H_{0}$ is a $k$-plane in $H^{\prime}$ containing $p$ and $q$ then $H_{0} \cap M$ is not connected. This proves the lemma.

Proof of the theorem. Let $M$ satisfy the conditions of the theorem and let $C(M)$ denote the convex hull of $M$. Let $p \in C(M)$. By the lemma every $k$-plane through $p$ intersects $M$. Since the intersection is acyclic it follows from Corollary 1.5 that $p \in M$, i.e. $M=C(M)$. This proves the theorem.

2.4. The following theorem gives homological conditions insuring that a set $M \subset E_{n}$ shall be a convex sphere $S_{n-1}$. (For the case $n=3$ see J. Schreier [6]).

THEOREM. Let $M$ be a compact subset of $E_{n}$. We suppose that 
(a) $H_{n-1}(M)=Z_{2}$ and $M$ is an irreducible carrier of the nonzero selement $\alpha$ of $H_{n-1}(M) .^{3}$

(b) There exists an integer $k, 0<k<n-1$, such that for every k-plane $T$ intersecting $M$

$$
H_{i}(T \cap M)=\left\{\begin{array}{lr}
0 & \text { for } i<k-1 \\
0 & \text { or } Z_{2} \text { for } i=k-1
\end{array}\right.
$$

Then $M$ is a convex $S_{n-1}$.

Proof. It is well known that (a) implies that $M$ disconnects $E_{n}$ into two sets and is the boundary of each. Let $G$ be the bounded component of $E_{n}-M$ and let $M^{*}=M \cup G$. Then

(i) $M^{*}$ is compact and $M=F r M^{*}$

Let $p \in G$ and let $T$ be a $k$-plane through $p$. We will prove (ii) $T \cap M^{*}$ is acyclic.

Since $T \cap M^{*}=(T \cap M) \cup(T \cap G)$ and $T \cap G \neq 0$ it follows that $T \cap M$ disconnects $T$. Therefore by (b)

$$
H_{i}(T \cap M)=\left\{\begin{array}{lll}
0 & \text { for } & i<k-1 \\
Z_{2} & \text { for } & i=k-1
\end{array}\right.
$$

It follows that $T-M$ has exactly two components; let the bounded component be $H$. Then $p \in H$ and we have $T \cap G=H$. Thus we have proved

(iv) $T \cap M^{*}$ is the union of $T \cap M$ and the bounded component $H$ of $T-T \cap M$.

Now, this implies that $\operatorname{Fr}\left(T \cap M^{*}\right) \subset T \cap M$ and we may consider the sequence

$$
H_{l}\left(F r\left(T \cap M^{*}\right)\right) \stackrel{i_{*}}{\longrightarrow} H_{l}(T \cap M) \stackrel{j_{*}}{\longrightarrow} H_{l}\left(T \cap M^{*}\right)
$$

where $i_{*}, j_{*}$ are induced by inclusions. By [4] $j_{*} i_{*}$ is onto, thus such is also $j_{*}$ and by (iii) this implies $H_{l}\left(T \cap M^{*}\right)=0$ for $l<k-1$. Since (iv) implies that $T \cap M^{*}$ does not disconnect $T$, also $H_{k-1}\left(T \cap M^{*}\right)=0$, which completes the proof of (ii).

Now, by the theorem mentioned in 2.1, (ii) implies that $M^{*}$ is starshaped with respect to every point of $G$. Let now $p \in M$ and $x \in M^{*}$. Let $p_{n} \in G, p_{n} \rightarrow p$, by the remark above segments $\overline{p_{n} x}$ are in $M^{*}$. Since $\overline{p_{n} x} \rightarrow \overline{p x}$ it follows that $\overline{p x} \subset M^{*}$ and thus $M^{*}$ is star-shaped with respect to every point. Thus $M^{*}$ is convex. Together with (i) this proves the theorem.

${ }^{3}$ i.e., for every proper compact subset $A \subset M \alpha$ is not in the image $H_{n-1}(A) \rightarrow H_{n-1}(M)$ (inclusion homomorphism). 
2.5. It is interesting to note that the following theorems, connected with the Borsuk-Ulam theorem (see e.g. [5]), follow easily from the results in $\S 1$.

$S_{n}$ will stand for the unit $n$-sphere in $E_{n+1}, \alpha: S_{n} \rightarrow S_{n}$ will denote the antipodal involution on $S_{n}$.

2.51. THEOREM. Let $f: S_{n} \rightarrow S_{n}$ be a continuous mapping and suppose that $f(x) \neq \alpha(x)$ for every $x \in S_{n}$. Then for some $x_{0} \in S_{n} \alpha f\left(x_{0}\right)=$ $f \alpha\left(x_{0}\right)$.

Proof. For every $x \in S_{n}$ let $a(x)=x+f(x)$ and let $H(x, \alpha(x))$ be the $n$-plane through 0 and perpendicular to the line through $x$ and $\alpha(x)$. Then $a(x)$ lies on a sphere of radius 1 and with center at $x$ and it follows from the assumption $f(x) \neq \alpha(x)$ that the points $a(x)$ and $a(\alpha(x))$ are distinct and lie in distinct components of $E_{n+1}-H(x, \alpha(x))$. Therefore the intersection $\Phi(x, \alpha(x))$ of the segment $a(x) a(\alpha(x))$ with $H(x, \alpha(x))$ is nonempty and $\mathscr{T}=\left\{P_{n}, E_{n}, \Phi\right\}$ is an acyclic family. Thus, by 1.3 , for some $x \Phi(x, \alpha(x))$ intersects also the orthogonal complement of $H(x, \alpha(x))$. It follows that for some $x, \Phi(x, \alpha(x))=0$. Then $0, a(x)$, $a(\alpha(x))$ are distinct and collinear. It is easy to see that this implies $\alpha a(x)=a(\alpha(x))$, i.e., $\alpha f(x)=f \alpha(x)$. Q.E.D.

By similar method one can obtain the following generalizations of 2.51 (proofs are omitted):

2.52. THEOREM. Let $F: S_{n} \rightarrow S_{n}$ be an acyclic upper semicontinuous: map and suppose that $F(x) \cap(\alpha(x))=0$. Then for some $x_{0}, \alpha\left(F\left(x_{0}\right)\right) \cap$ $F\left(\alpha\left(x_{0}\right)\right) \neq 0$.

2.53. THEOREM. Let $\beta$ be the antipodal involution in the bundle. $B$ of unit vectors tangent to $S_{n}$. Let $F: S_{n} \rightarrow B$ be a multivalent acyclic cross-section. Then for some $x_{0} \in S_{n}, \beta F\left(x_{0}\right) \cap F\left(\alpha\left(x_{0}\right)\right) \neq 0$.

2.6. In the following two theorems $M$ will denote a differentiable. $(n-1)$-manifold in $E_{n}$. If $p \in M$ then $T(p)$ will denote the tangent. plane to $M$ at $p$. $T \| T^{\prime}$ will mean that $T$ is parallel to $T^{\prime}$.

2.61. THEOREM. Let $f: M \rightarrow E_{n}$ be a continuous mapping such that if $T(p)$ is parallel to $T\left(p^{\prime}\right)$ then the vector $\overrightarrow{p f(p)}$ is not zero and parallel. to $\overrightarrow{p^{\prime} f\left(p^{\prime}\right)}$. Then for some $\overrightarrow{p_{0} \in M p_{0} f\left(p_{0}\right)}$ is perpendicular to $T\left(p_{0}\right)$.

Proof. It is known that for every $(n-1)$-plane $H$ through the origin there exists a $p \in M$ such that $H \| T(p)$. Let then $\Phi(H)$ be the endpoint of the unit vector parallel to $\overrightarrow{p f(p)}$. By our assumption about $f$, the 
family $\mathscr{F}=\left\{P_{n-1}, E_{n}, \Phi\right\}$ is well-defined and, obviously, acyclic. The theorem follows then from 1.4 .

Theorem 2.61 can be again generalized to acyclic multivalent maps $f: M \rightarrow E_{n}$. Instead, we prove:

2.62. THEOREM. Let $f: M \rightarrow E_{n}$ be an immersion such that if $T(p) \| T\left(p^{\prime}\right)$ then $T(f(p)) \| T\left(f\left(p^{\prime}\right)\right)$. Then for some $p_{0} \in M, T\left(p_{0}\right) \| T\left(f\left(p_{0}\right)\right)$.

Proof. We suppose $M$ oriented and a field of normal unit vectors given on $M$. This defines a field of unit vectors normal to $f(M)$ and we define the family $\mathscr{F}=\left\{P_{n}, E_{n}, \Phi\right\}$ as follows: Let $H(x)$ represent a point $x \in P_{n}$, there exists a point $p \in M$ such that $T(p) \| H(x)$. We define $\Phi(x)=$ the endpoint of the unit vector normal to $f(M)$ at $f(p)$. By our assumption about $f$ the family $\mathscr{F}$ is well-defined. Then the theorem follows from 1.4 .

\section{REFERENCES}

1. G. Aumann, On a topological characterization of compact convex point sets, Ann. of Math., 37 (1936), 443.

2. E. G. Begle, The Vietoris mapping theorem for bicompact spaces, Ann. of Math., 51 (1950), 534.

3. K. Borsuk and A. Kosinski, Families of acyclic compacta in Euclidean $n$-space, Bull. Acad. Pol. Sci. C1. III, 3 (1955), 293.

4. - On connections between the homology properties of a set and of its frontier, Bull. Acad. Pol. Sci. Cl. III, 4 (1956), 331.

5. G. Hirsch, Un théorème sur les transformations des sphères, Acad. Roy. Belgique, Bull. Cl. Sci., 32 (1946), 394.

6. J. Schreier, Über Schnitte Konvexen Flächen, Bull. Int. Ac. Pol. Serie A (1933), 155.

7. S. K. Stein, Continuous choice function and convexity, Fund. Math., 45 (1958), 182.

8. H. Steinhaus, Quelques applications des principes topologiques à la géométrie des corps convexes, Fund. Math., 41 (1955), 284. 



\section{PACIFIC JOURNAL OF MATHEMATICS}

\section{EDITORS}

Ralph S. Phillips

Stanford University

Stanford, California

M. G. Arsove

University of Washington

Seattle 5, Washington
A. L. Whiteman

University of Southern Californla

Los Angeles 7, California

Lowell J. Paige

University of California

Los Angeles 24, California

\section{ASSOCIATE EDITORS}

E. F. BECKENBACH

D. DERRY

H. L. ROYDEN

E. G. STRAUS

T. M. CHERRY

M. OHTSUKA

E. SPANIER

F. WOLF

\section{SUPPORTING INSTITUTIONS}

UNIVERSITY OF BRITISH COLUMBIA

STANFORD UNIVERSITY

CALIFORNIA INSTITUTE OF TECHNOLOGY

UNIVERSITY OF CALIFORNIA

MONTANA STATE UNIVERSITY

UNIVERSITY OF TOKYO

UNIVERSITY OF UTAH

UNIVERSITY OF NEVADA

NEW MEXICO STATE UNIVERSITY

OREGON STATE UNIVERSITY

UNIVERSITY OF OREGON

OSAKA UNIVERSITY

WASHINGTON STATE UNIVERSITY

UNIVERSITY OF WASHINGTON

UNIVERSITY OF SOUTHERN CALIFORNIA

AMERICAN MATHEMATICAL SOCIETY CALIFORNIA RESEARCH CORPORATION SPACE TECHNOLOGY LABORATORIES NAVAL ORDNANCE TEST STATION 


\section{Pacific Journal of Mathematics}

\section{Vol. 12, No. $1 \quad$ January, 1962}

Jonathan L. Alperin, Groups with finitely many automorphisms $\ldots \ldots \ldots \ldots \ldots \ldots \ldots \ldots$

Martin Arthur Arkowitz, The generalized Whitehead product ................ 7

John D. Baum, Instability and asymptoticity in toplogical dynamics . . . . . . . . . . 25

William Aaron Beyer, Hausdorff dimension of level sets of some Rademacher series .... $\quad 35$

Frank Herbert Brownell, III, A note on Cook's wave-matrix theorem . . . . . . . . . . . . . 47

Gulbank D. Chakerian, An inequality for closed space curves ................. 53

Inge Futtrup Christensen, Some further extensions of a theorem of Marcinkiewicz ....... 59

Charles Vernon Coffman, Linear differential equations on cones in Banach spaces . . . . . 69

Eckford Cohen, Arithmetical notes. III. Certain equally distributed sets of integers . . . . . 77

John Irving Derr and Angus E. Taylor, Operators of meromorphic type with multiple poles

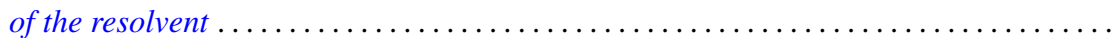

Jacob Feldman, On measurability of stochastic processes in products space .............

Robert S. Freeman, Closed extensions of the Laplace operator determined by a general class of boundary conditions, for unbounded regions ......................

Robert E. Fullerton, Geometric structure of absolute basis systems in a linear topological

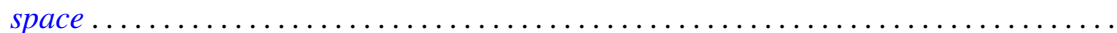

Dieter Gaier, On conformal mapping of nearly circular regions

Andrew Mattei Gleason and Hassler Whitney, The extension of linear functionals defined

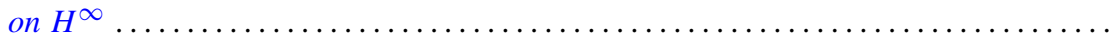

Seymour Goldberg, Closed linear operators and associated continuous linear

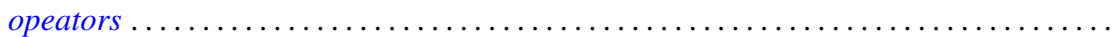

Basil Gordon, Aviezri Siegmund Fraenkel and Ernst Gabor Straus, On the determination of sets by the sets of sums of a certain order

Branko Grünbaum, The dimension of intersections of convex sets. .

Paul Daniel Hill, On the number of pure subgroups

Robert Peter Holten, Generalized Goursat problem . .

Alfred Horn, Eigenvalues of sums of Hermitian matrices ...........

Henry C. Howard, Oscillation and nonoscillation criteria for

$$
y^{\prime \prime}(x)+f(y(x)) p(x)=0
$$

Taqdir Husain, $S$-spaces and the open mapping theorem ...

Richard Eugene Isaac, Markov processes and unique stationary probability measures ...

John Rolfe Isbell, Supercomplete spaces ....................

John Rolfe Isbell, On finite-dimensional uniform spaces. II .........

N. Jacobson, A note on automorphisms of Lie algebras ..............

Antoni A. Kosinski, A theorem on families of acyclic sets and its applications

Marvin David Marcus and H. Minc, The invariance of symmetric functions of singular values...

Ralph David McWilliams, A note on weak sequential convergence.

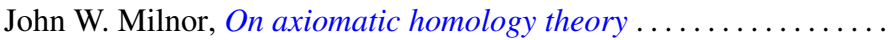

Victor Julius Mizel and Malempati Madhusudana Rao, Nonsymmetric projections in

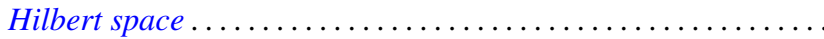

Calvin Cooper Moore, On the Frobenius reciprocity theorem for locally compact

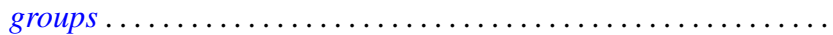

Donald J. Newman, The Gibbs phenomenon for Hausdorff means . 